\title{
Apoplejía tumoral hipofisaria posquirúrgica. A propósito de dos casos
}

\author{
J. González-Tortosa y M. Poza-Poza
}

Servicio de Neurocirugía. Hospital Universitario Virgen de la Arrixaca. Murcia.

\section{Resumen}

Se presentan dos casos de macroadenomas no funcionantes grandes que, tras su extirpación parcial por vía transesfenoidal, sufrieron un infarto del resto tumoral residual, con resultado de aumento de su volumen, compresión de las estructuras vecinas y deterioro neurológico de los pacientes. Ambos fueron reintervenidos por vía transcraneal para conseguir la extirpación completa de sus tumores. Se revisa la bibliografía en busca de las claves para prevenir esta complicación.

PALABRAS CLAVE: Adenoma hipofisario. Apoplejía hipofisaria. Cirugía transesfenoidal. Complicación.

Postsurgical pituitary apoplexy. Report of two cases

\section{Summary}

We report two cases of large macroadenomas that, after a transsphenoidal partial resection, suffered necrosis and swelling of the residual tumor, with increase of its volume, compression of neighboring structures and neurological deterioration. The literature is reviewed looking for possible pathophysiological mechanism and prevention.

KEY WORDS: Pituitary adenoma. Pituitary apoplexy. Transsphenoidal surgery. Complication.

\section{Introducción}

Las complicaciones de la cirugía transesfenoidal de los adenomas hipofisarios están bien estudiadas en la literatura médica ${ }^{2,3,8,11,12}$. Sin embargo, la apoplejía de los restos tumorales en el posoperatorio inmediato, es un hecho que apenas ha recibido atención en la bibliografía. Con la presentación de estos dos casos, queremos llamar la atención

Recibido: 17-12-08. Aceptado: 24-04-09 sobre esta posibilidad y analizar las claves para evitarla, ya que conlleva una grave morbilidad y mortalidad para el paciente.

\section{Paciente 1}

Varón de 50 años, con macroadenoma hipofisario de $4,38 \mathrm{~cm}$ de diámetro vertical, que destruye silla turca y ocupa el seno esfenoidal. La extensión intracraneal comprime el tercio anterior del tercer ventrículo hasta las inmediaciones de los agujeros de Monro, sin provocar hidrocefalia. La cúpula del adenoma no es enteramente uniforme, adoptando una ligera forma trilobulada (Fig.1 y 2).

El paciente presentaba una hemianopsia temporal en ojo izquierdo (OI) con motilidad ocular normal. En los estudios hormonales existía un déficit de testosterona y un moderado aumento de prolactina (PRL).

Se intervino quirúrgicamente mediante un abordaje transesfenoidal clásico: sublabial transeptal. El tumor presentaba las porciones centrales friables, pero era muy sólido y sangrante en las periféricas. Se extirpó todo el tumor que ocupaba el seno esfenoidal, silla turca y gran parte de su porción supraselar, hasta donde alcanzaban las curetas. Sin embargo, el diafragma de la silla turca no descendió al campo quirúrgico visible. Se logró una buena hemostasia con $\mathrm{H}_{2} \mathrm{O}_{2}$ y celulosa oxidada. Se rellenó el seno esfenoidal y la silla turca con grasa autóloga y, para sostenerla in situ, se realizó una plastia de la cara anterior del seno esfenoidal con hueso vómer del propio paciente.

El examen anatomo-patológico confirmó un adenoma hipofisario.

Cuando el enfermo despertó de la anestesia, se detectó una amaurosis completa de OI, conservando la visión sólo en el cuadrante visual nasal superior del ojo derecho (OD), así como una parálisis del VI y III pares izquierdos. La resonancia magnética $(\mathrm{RM})$ urgente que se le practicó, mostró la presencia de un nódulo tumoral supraselar aumentado de tamaño, con edema y restos de sangre (Fig.3).

Se reintervino al paciente de urgencia, mediante una craneotomía pterional derecha. El tumor comprimía las 


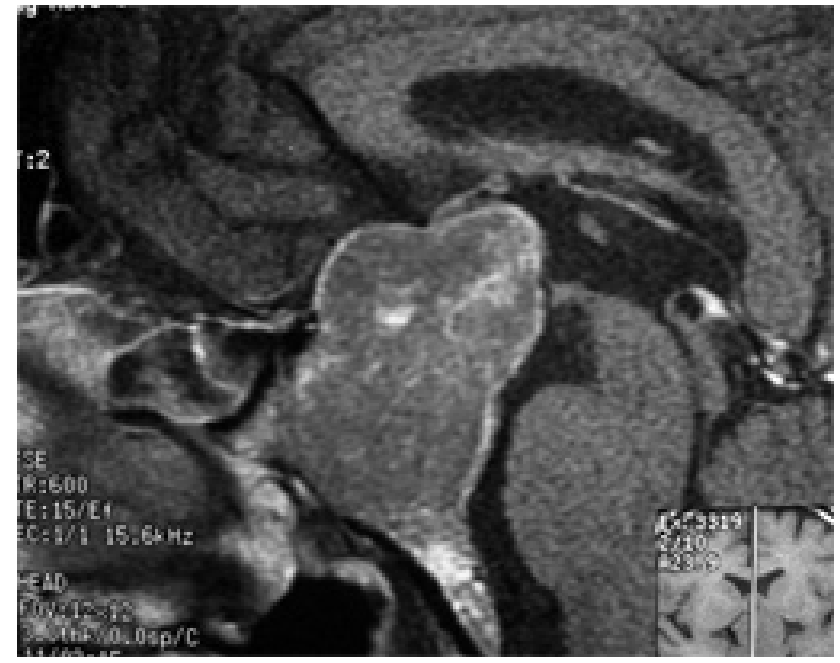

Figura 1. Caso $n^{o}$ 1. RM preoperatoria sagital $+C$. El tumor ocupa todo el seno esfenoidal, ha destruido la silla turca y su cúpula -trilobulada-comprime la parte anterior del III ventrículo. Se aprecia la indentación que le provoca el diafragma selar.

vías ópticas, en especial el nervio óptico izquierdo que aparecía acintado sobre una cápsula tumoral de color violáceo oscuro, rechazando hacia atrás el quiasma óptico postfijado. En el interior del tumor no se encontró hematoma, sino tejido tumoral sólido y de color violáceo, que se extirpó junto con la cápsula tumoral, previa separación de las vías ópticas. Se conservó in situ el injerto de grasa dejado en el fondo de la silla turca y seno esfenoidal en la primera intervención.

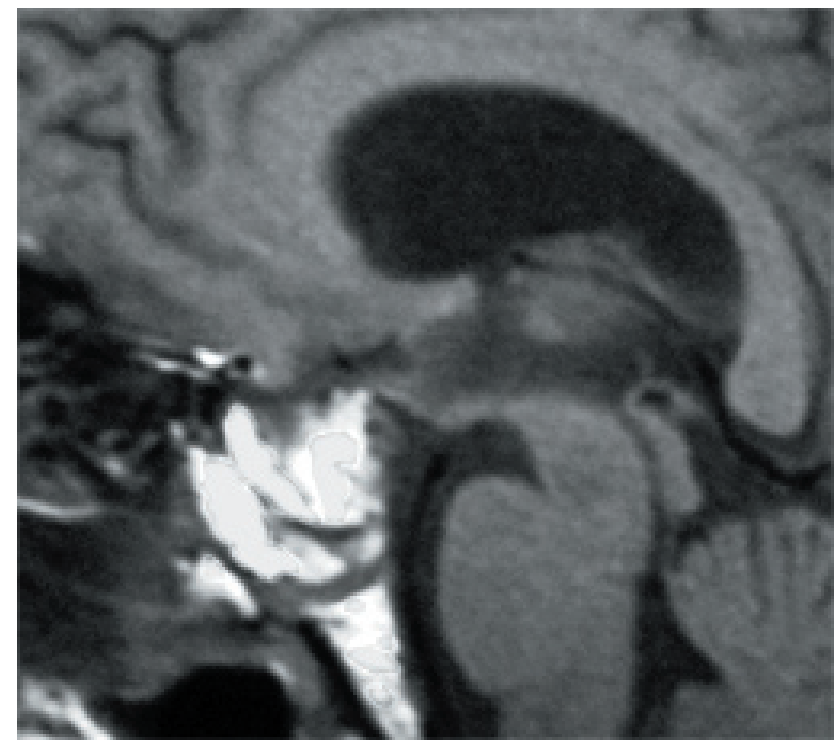

Figura 4. Caso $n^{\circ}$ 1. RM T1 sagital después de la reintervención. La cisterna supraselar está libre de tumor. En la silla turca y seno esfenoidal se aprecia la grasa autóloga con la que se rellenaron en el abordaje transesfenoidal.

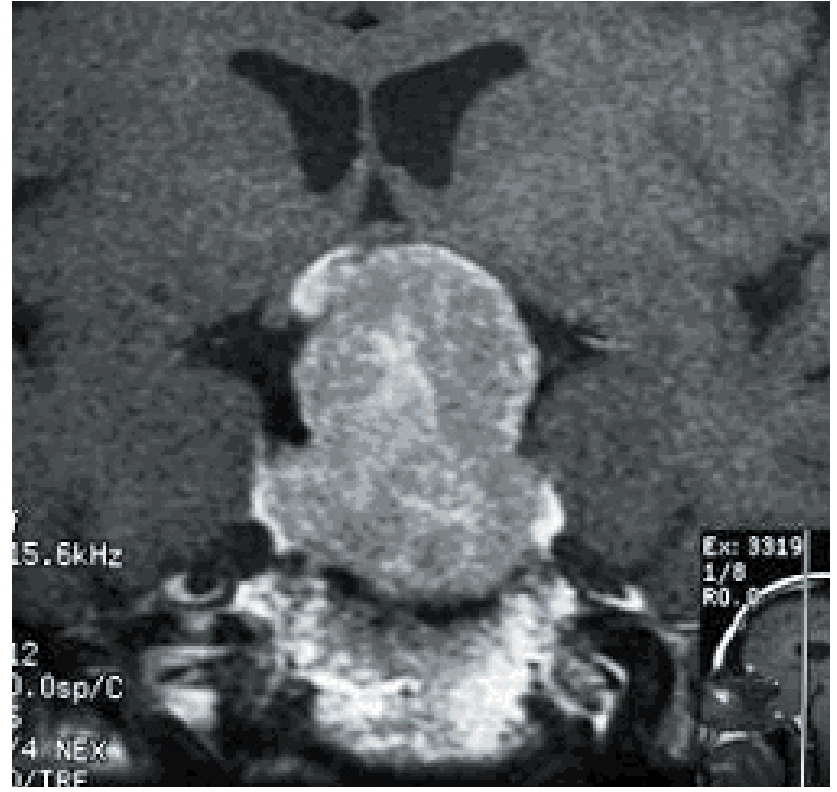

Figura 2. Caso $n^{o}$ 1. RM preoperatoria coronal + contraste. En esta proyección el tumor comprime gravemente las vías ópticas y tiene también una pequeña indentación diafragmática.

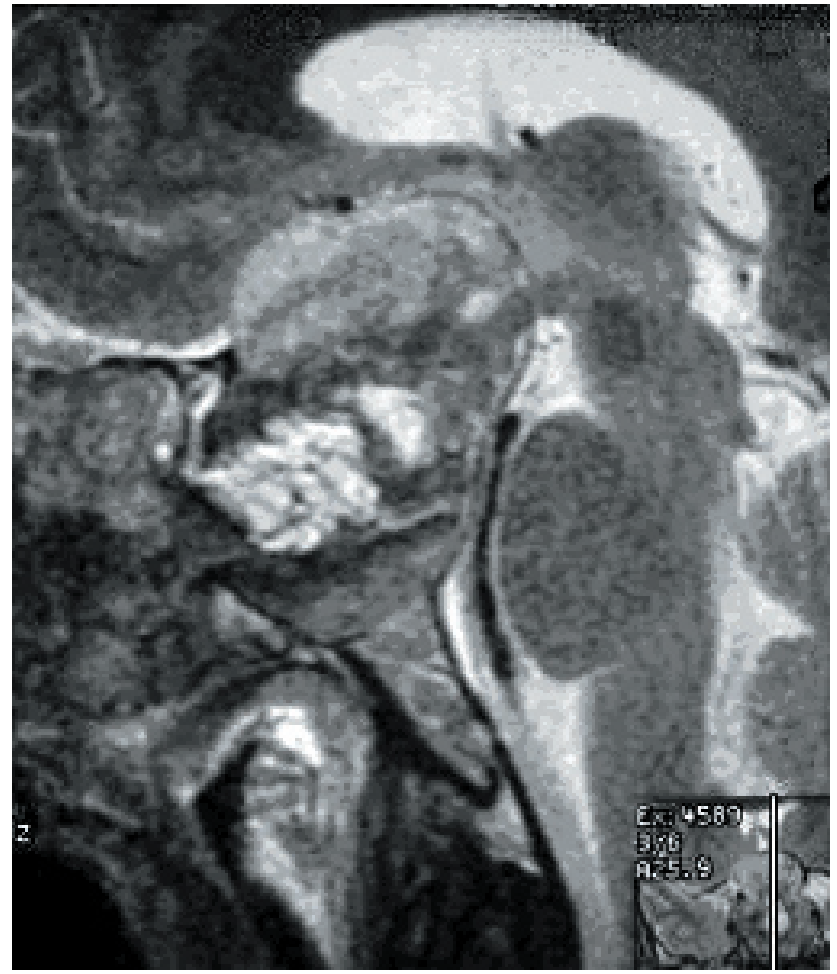

Figura 3: Caso $n^{\circ}$ 1. RM T2 sagital tras la intervención por vía transesfenoidal. Seno esfenoidal y silla turca están ocupados por material quirúrgico y restos hemáticos. El nódulo tumoral supraselar edematizado ha aumentado de volumen y comprime, más que en el preoperatorio, al tercer ventrículo. 
El paciente sobrevivió con graves secuelas visuales. $\mathrm{Al}$ año de evolución presentaba pérdida de todo el campo visual por OI y de los campos temporales y nasal superior del OD, por donde podía distinguir dedos a 3 metros. Al déficit de testosterona, se había añadido un déficit suprarrenal, siendo el resto de las hormonas normales. En la RM se podía apreciar la desaparición del tumor con la presencia de la grasa de los injertos rellenando la silla turca y seno esfenoidal (Fig. 4).

\section{Paciente 2}

Varón de 61 años con un adenoma hipofisario de 4,1 $\mathrm{cm}$ de diámetro vertical, que destruía la silla turca e invadía seno esfenoidal, ocupándolo por completo. Así mismo invadía de forma moderada el seno cavernoso derecho y se extendía por la cisterna supraselar, desplazando las vías ópticas y comprimiendo el tercio anterior del tercer ventrículo.

El examen oftalmológico reveló una palidez de ambas papilas ópticas. Agudeza visual $<0,1$ en OD y 0,7 en OI. Hemianopsia bitemporal, a la que se añadía una cuadrantanopsia nasal en OD. La motilidad ocular era normal.

Los estudios hormonales basales fueron normales, aunque las hormonas tiroideas se encontraban en el límite inferior de la normalidad. No se determinó el eje somatotrópico.

Se intervino quirúrgicamente por vía transesfenoidal, mediante un abordaje unilateral endonasal transeptal izquierdo. El tumor abultaba desde el seno esfenoidal, estando cubierto sólo por mucosa al estar erosionado el hueso. Presentaba unas porciones centrales friables, pero era sólido y muy consistente en las periféricas. Se extirparon sus porciones esfenoidal y selar, así como la extensión supraselar accesible. La neumoencefalografía intraoperatoria, realizada a través de un catéter lumbar previamente colocado, mostró la presencia de aire rellenando la cisterna supraselar, confirmando la impresión del cirujano de que el diafragma selar había caído al campo quirúrgico. Se rellenó el lecho quirúrgico con fascia lata, músculo y grasa autóloga, sellando el seno esfenoidal con un trozo de cartílago nasal.

El examen anatomo-patológico confirmó el adenoma hipofisario.

En el postoperatorio inmediato, el paciente desarrolló, de forma progresiva y en este orden: amaurosis derecha, parálisis del III par derecho a la que siguió una parálisis del III par izquierdo y, por último una pérdida de agudeza visual adicional en OI. Se practicó una RM postoperatoria urgente que puso en evidencia la presencia de un nódulo tumoral supraselar aumentado de volumen, con signos de edema tisular y restos hemorrágicos en el lecho quirúrgico

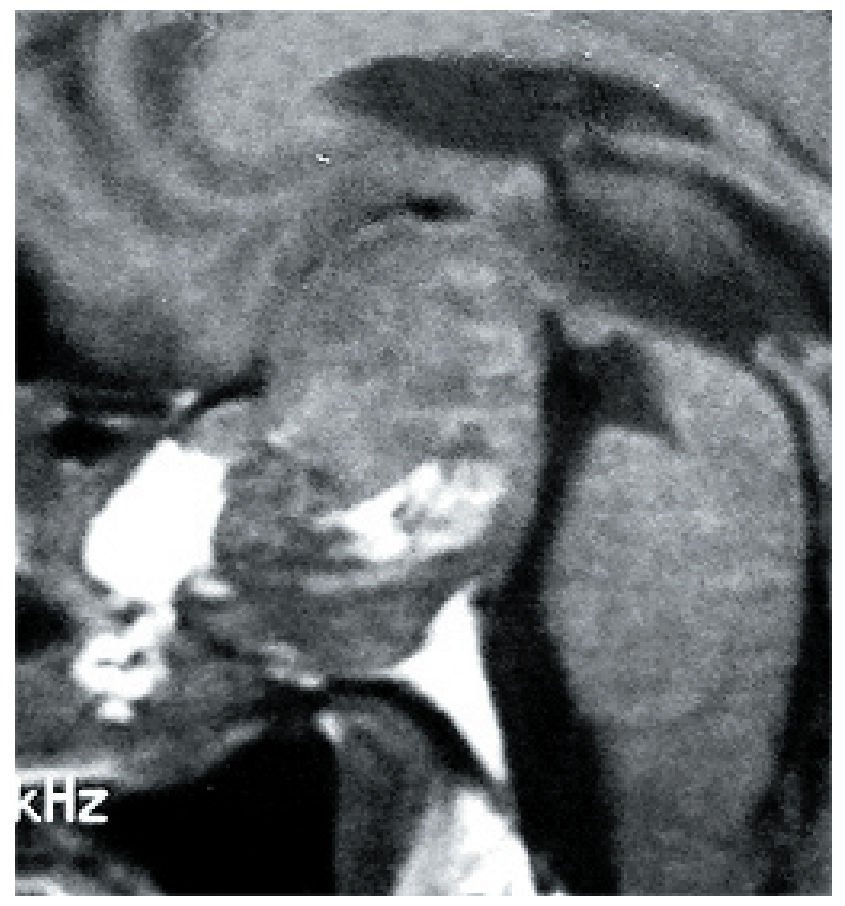

Figura 5. RM T1 postoperatoria del segundo paciente. Nódulo tumoral residual supraselar, con signos de edema y aumentado de volumen, comprime quiasma y porción anterior del tercer ventrículo. En la silla turca y seno esfenoidal existen restos hemáticos y de material quirúrgico. La grasa autóloga ha sido desplazada hacia delante por la presión a nivel del lecho quirúrgico.

\section{(Fig 5).}

Se reintervino urgentemente por vía pterional derecha: Se extirpó el nódulo tumoral supraselar, de color violáceo, con su cápsula, separándolo del quiasma e hipotálamo. No existía un hematoma organizado intratumoral. El nervio óptico derecho se encontraba gravemente comprometido por el tumor y acintado. El nervio óptico izquierdo estaba estirado hacia un quiasma óptico muy desplazado hacia atrás.

El examen del tumor extraído mostró la proliferación celular conocida, ahora en gran parte necrótica.

El paciente sobrevivió con graves secuelas visuales: amaurosis en OD; cuadrantanopsia nasal inferior en OI con agudeza visual por este ojo normal. Desde el punto de vista hormonal presenta un panhipopituitarismo que requiere tratamiento sustitutivo.

\section{Discusión}

El aumento de volumen del tumor pituitario residual tras su resección quirúrgica parcial ha sido raramente publicado y desconocemos si su incidencia está subestimada. Decker y Chalif ${ }^{4}$ en 1991, presentaron dos casos 
de tumores grandes que comprometían la circulación del líquido cefalorraquídeo a nivel del tercer ventrículo, provocando hidrocefalia moderada. Tras ser sometidos a resección parcial por vía transesfenoidal, sufrieron deterioro neurológico por aumento de la hidrocefalia, siendo el mecanismo más probable el aumento del volumen del tumor residual. Lee y Railly ${ }^{9}$ en 2001, publicaron un caso de adenoma grande, de $5 \mathrm{~cm}$ de diámetro vertical, que comprimía el tercer ventrículo y tras su resección parcial por vía transesfenoidal, el paciente sufrió deterioro neurológico y tuvo que ser reoperado de urgencia por vía transcraneal, encontrando un infarto hemorrágico del tumor residual, sin hematoma significativo. Cappabianca ${ }^{2}$, en 2002, en un macroadenoma paninvasivo extirpado de manera parcial mediante endoscopia nasal, relató el empeoramiento y muerte del paciente debido a un edema de la lesión residual con compresión del tercer ventrículo y tronco cerebral. Ahmad y col ${ }^{1}$, publicaron en 2005 tres pacientes con tumores también grandes que, tras su extirpación parcial por vía transesfenoidal, sufrieron deterioro neurológico en el inmediato postoperatorio por hemorragia intratumoral en la lesión residual, falleciendo los tres casos pese a la reintervención urgente por vía transcraneal. En el mismo año, Kurschel y col$^{7}$ dieron a conocer un caso similar en el que la apoplejía provocó una compresión de las arterias carótidas con infarto cerebral bilateral en el territorio de las arterias cerebral anterior y media, con resultado de fallecimiento del enfermo.

La apoplejía de los restos del adenoma, con aumento de volumen tras su extirpación parcial y compresión de las estructuras colindantes, también se ha publicado en 4 pacientes con tumores grandes abordados por vía transcraneal ${ }^{1,5,9}$, con resultado de muerte en 3 de ellos.

El mecanismo por el que se producen estos infartos del resto tumoral dejado in situ, con edema secundario $\mathrm{y}$ aumento de su volumen, a veces con hemorragia, no es conocido. En todos los casos publicados se trataba de tumores muy grandes. Se ha sugerido que la manipulación quirúrgica provoca un edema agudo en el tumor residual ${ }^{4}$ y que este aumento de volumen produce, además, la compresión de las arterias hipofisarias superiores y de los vasos porta, con la consiguiente isquemia y necrosis ${ }^{9,10}$. También se sugiere que el descenso parcial del resto tumoral tras la cirugía transesfenoidal, coarte estos vasos con el mismo efecto. Es posible que la porción supraselar del tumor reciba parte de su nutrición vascular a través de la porción inferior que se extirpa, provocando su necrosis. Kurschel y $\mathrm{col}^{7}$, publicaron un caso en el que el paciente había sufrido durante la intervención un episodio de caída de la tensión arterial sistólica de 140 a $80 \mathrm{~mm}$ de $\mathrm{Hg}$ durante 20 minutos, lo que pudiera haber influido en el desarrollo de la apoplejía del resto tumoral no extirpado.

En los abordajes transcraneales, se ha implicado la interrupción de las ramas arteriales carotídeas hacia el tumor $^{5}$ o la interrupción del drenaje venoso. Es posible que el drenaje venoso del tumor supraselar se realice hacia los senos cavernosos a través de vasos tumorales que se extirpan con el tumor a nivel de la silla turca en el abordaje transesfenoidal.

Las consecuencias de esta complicación pueden resultar fatales. Nuestros dos pacientes quedaron con secuelas visuales graves y con deterioro hormonal. En todos los casos se trataba de tumores grandes, por encima de los $4 \mathrm{~cm}$ de diámetro, con compromiso visual previo a la cirugía y compresión, en mayor o menor grado, del tercer ventrículo.

Las indicaciones de la cirugía transcraneal de los adenomas hipofisarios están bien establecidas ${ }^{8}$, e incluyen los tumores con una extensión supraselar grande y componente intraselar pequeño, los que tienen forma en reloj de arena con cuello pequeño a nivel del diafragma selar y los que se extienden por detrás de la silla turca, teniendo ésta el dorso conservado, o los que lo hacen hacia fosa anterior o media. Cuando se plantea el abordaje combinado, transcraneal y transesfenoidal, se recomienda hacer siempre el transesfenoidal primero ${ }^{8}$.

Nuestra política, desde que tuvimos esta experiencia, ha sido la de abordar tumores de estas características -alteración visual grave previa a la cirugía, tumor de $>4 \mathrm{~cm}$ de diámetro vertical, con mayor extensión supraselar que sellar y que comprime tercer ventrículo, aunque el tumor no tenga forma de reloj de arena y su cúpula sea uniformepor vía transcraneal en primera instancia, con el objetivo de intentar la liberación completa de las vías ópticas y tercer ventrículo del tumor supraselar. Tras más de 150 abordajes transesfenoidales realizados con posterioridad siguiendo esta política, no hemos tenido ninguna complicación similar.

Actualmente estamos empleando técnicas de endoscopia en la cirugía transesfenoidal, con las que se obtiene una mejor visualización del tumor supraselar que, con el microscopio óptico, cae fuera del campo quirúrgico directo. Sin embargo, encontramos la misma dificultad para extraer la porción supraselar de los tumores sólidos, que está alejada del alcance de la instrumentación, si espontáneamente no desciende tras la escisión de la porción esfenoidal y selar. El caso publicado por Cappabianca es una muestra de ello ${ }^{2}$. El problema se agrava si el tumor es fibroso. Para aminorar esta dificultad, se puede realizar un abordaje endoscópico ampliado, pero el procedimiento ya no es tan mínimamente invasivo y nos enfrentamos al problema de la fístula de lcr, para cuya prevención se está imponiendo la práctica de colgajos vascularizados de mucosa nasal que se rotan para sellar el defecto en la base de cráneo ${ }^{6}$. Con la suficiente experiencia puede ser una alternativa, pero nosotros seguimos prefiriendo la pequeña craneotomía pterional 
para este tipo de pacientes.

\section{Bibliografía}

1. Ahmad, F.U., Pandey, P., Mahapatra, A.K.: Post operative 'pituitary apoplexy' in giant pituitary adenomas: a series of cases. Neurology India 2005; 53: 326-328.

2. Cappabianca, P., Cavallo, L.M., Colao, A.M., Divitiis, E.: Surgical complications associated with the endoscopio endonasal transsphenoidal approach for Pituitary adenomas. J Neurosurg 2002; 97: 293-298.

3. Ciric, I., Ragin, A., Baumgartner, C., Pierce, D.: Complications of Transsphenoidal Surgery: Results of a National Survey, Review of the Literature, and Personal Experience. Neurosurg 1997; 40: 225-237.

4. Decker, R.E., Chalif, D.J.: Progressive coma after the trassphenoidal descompression of a Pituitary adenoma with marked suprasellar extensión: Report of two cases. Neurosurgery $1991 ; 28$ : 154-158.

5. Goel, A., Deogaonkar, M., Desai, D.K.: Fatal postoperative 'pituitary apoplexy': its cause and management. Brit J Neurosurg 1995; 9: 37-40.

6. Hadad, G., Bassagasteguy, L., Carrau, R.L., Mataza, J.C., Kassam, A., Snyderman, C.H., Mintz, A.: A novel reconstructive technique after endoscopio expandes endonasal

\section{Comentarios al trabajo Apoplejía tumoral hipofisaria posquirúrgica de J. González Tortosa y M. Poza.}

El deterioro de la función visual o la aparición de alteración neurológica, tanto de la conciencia como de los pares craneales oculomotores, en el postoperatorio inmediato de un adenoma de hipófisis es un hecho bien conocido por los cirujanos que se dedican al tratamiento quirúrgico de estos pacientes.

La causa parece estar claramente relacionada con el fenómeno quirúrgico y el proceso fisiopatológico podría explicarse por las mismas condiciones que llevan a deterioro neurológico tras resección parcial de un meningioma o un glioma; aunque lógicamente en un contexto anatómico diferente.

De la bibliografía revisada por los Drs. GonzalezTortosa y Poza se puede deducir que es un fenómeno que no está en relación directa con la experiencia, la vía, la técnica quirúrgica, la pericia o habilidad del cirujano. approaches: vascular pedicle nasoseptal flap. Laryngoscope 2006; 116:m1882-1886.

7. Kurschel, S., Leber, K.A., Scarpatetti, M., Roll, P.: Rare fatal vascular complication of transsphenoidal surgery. Acta Neurochir (Wien) 2005; 147: 321-325.

8. Landolt, A.M.: Transsphenoidal surgery of Pituitary tumors: Its pitfalls and complications. Prog Neurol Surg. Basel. Karger 1990; 13: 1-30.

9. Lee, G.Y.F., Reilly, P.L.: Pituitary surgery complicated by haemorrhagic necrosis of residual tumor. Pituitary 2001; 4 : 279-82.

10. Rovit, R.L., Fein, J.M.: Pituitary apoplexy: a review and reappraisal. J Neurosurg 1972; 37: 280-288.

11. Sudhakar, N., Vafidis, J.A.: Complications after transsphenoidal surgery: our experience and review of the literature. Brit J Neurosurg 2004; 18: 507-512.

12. Zada, G., Nelly, D.F., Cohan, P., Wang, C., Swerdloff, R.: Endonasal transsphenoidal approach for Pituitary adenomas and other sellar lesions: an assessment of efficacy, safety, and patients impressions. J Neurosurg 2003; 98: 350358.

González-Tortosa, J.; Poza-Poza, M.: Apoplejía tumoral hipofisaria posquirúrgica. A propósito de dos casos. Neurocirugía 2010; 21: 30-36.
Todos tenemos experiencias personales de esta situación si hemos operado suficiente número de pacientes.

Probablemente podemos hablar de factores que predisponen a este fenómeno y dentro de ellos a factores evitables y no evitables.

Por factores no evitables entendería aquellos que suceden durante la resección de tumores presumiblemente resecables en un solo abordaje quirúrgico, como los dos ejemplos de los autores, a excepción de que en el estudio de $\mathrm{RMN}$, la secuencia $\mathrm{T}_{2}$ nos sugiera que estamos ante un tumor fibroso (imagen tumoral de franca hiposeñal (en $\mathrm{T}_{2}$ ) o tumores excesivamente hipercaptantes de contraste.

Por factores evitables entendería todos aquéllos ya descritos en los años 80 por los pioneros modernos de la cirugía transesfenoidal y que el artículo de A.M. Landolt 
explica con detalle.

Soy de la opinión de los autores en que ni el catéter lumbar, ni las maniobras de Valsalva o el endoscopio resuelven el problema de la resección completa de los adenomas en cualquiera de sus formas, aunque desde el punto de vista teórico el endoscopio sería una herra-
2010; 21:30-36

mienta más ventajosa que el microscopio en el manejo de adenomas duros y ricamente vascularizados.

\author{
JJ Acebes \\ Neurocirujano \\ L'Hospitalet de Llobregat
}

$* * * * * * * * * * * * * * * * * * * * * * * * * * * * * * * * * *$

Muchos pacientes intervenidos de macroadenomas hipofisarios grandes con resecciones parciales desarrollan cambios hemorrágicos o isquémicos en los restos tumorales como demuestran los controles postquirúrgicos habituales con TC o RM. Probablemente, dichos cambios estén producidos por cambios en la irrigación del parénquima tumoral residual en el que pueden intervenir la devascularización quirúrgica, los cambios en la microcirculación derivados de variaciones de presión en el sistema arterial y venoso hipofisarios o el descenso parcial de los restos producidos por la manipulación quirúrgica y la descompresión. Sin embargo, es excepcional que estas alteraciones lleguen a tal grado que sean responsables de deterioro neurológico progresivo del paciente en el postoperatorio inmediato.

Los autores presentan los casos de dos pacientes con esta complicación en los que fue necesaria una reintervención por un vía pterional para detener el deterioro neurológico. Así mismo, revisan la bibliografía encontrando únicamente 8 casos de apoplejía de restos tumorales tras una cirugía transesfenoidal y 4 tras una vía transcraneal, llamando la atención sobre su existencia, su escasa incidencia y su grave pronóstico, y dando unas pautas para prevenir su aparición.

Los autores proponen operar tumores hipofisarios de más de 4 centímetros de diámetro cráneo-caudal con importante compromiso visual inicialmente por una craneotomía aunque no tengan forma de reloj de arena para evitar la aparición de esta complicación. En nuestro centro, estos tumores son abordados de forma inicial por vía transesfenoidal porque pensamos que es una vía con menor morbilidad frente a la craneotomía convencional, sobre todo en el paciente mayor, y que consigue una adecuada descompresión de la vía visual. Aunque nunca se nos ha presentado esta complicación, su excepcionalidad hace que, de momento, no nos planteemos modificar nuestra conducta. La vía transcraneal la reservamos para tumores que debido a su dureza (fibrosos) o a otras causas no han podido resecarse por vía transesfenoidal de forma satisfactoria, o inicialmente en aquellos tumores grandes con marcada forma de reloj de arena y mucho mayor componente supraselar que intraselar.

En cuanto a la forma de tratamiento propuesta por los autores cuando se presenta esta complicación, estamos de acuerdo en que la vía transcraneal (pterional) es la que mejores beneficios puede aportar al paciente en situación de deterioro neurológico progresivo.

$$
\begin{array}{r}
\text { Adolfo de la Lama } \\
\text { Neurocirujano } \\
\text { Complejo Hospitalario Universitario de Vigo }
\end{array}
$$

$* * * * * * * * * * * * * * * * * * * * * * * * * * * * * * * * * *$

Con el desarrollo y sofisticación actual de las técnicas de neuroimagen y la mejoría de la atención sanitaria afortunadamente los tumores hipofisarios suelen diagnosticarse en el rango de micro, meso o adenomas intraselares, siendo cada vez más infrecuentes las lesiones gigantes, como las que nos presentan González-Tortosa y Poza en este artículo. Sin embargo, su manejo supone siempre uno de los retos neuroquirúrgicos fundamentales en lo que a patología selar se refiere. La magnitud de la lesión, su vascularización aberrante, el grave compromiso paratu- moral y la disfunción hipofisaria que acompaña a estos tumores condiciona importante morbilidad quirúrgica además de malos resultados posteriores con persistencia de tejido residual abundante e importantes secuelas neurológicas sobre todo en vía óptica. Si a esto se añaden datos de sangrado en dicho tejido residual, el pronóstico es infausto como bien comentan los autores. Su experiencia, aunque no publicada, es amplia y muy valiosa en cuanto a sugerir abordaje transcraneal como primer paso en el acceso quirúrgico de estas importantes lesiones. Se echa de 
menos un estudio endocrinológico más exhaustivo, ya que es extraño que con el tamaño tumoral sólo tengan déficits aislados y no panhipopituitarismo, que suele ser lo habitual en macroadenomas paninvasivos como los presentados y el hipoadrenalismo agudo en la cirugía debería estar contemplado para evitar episodios bruscos de hipotensión que predispondrían al sangrado como en algún caso que comentan. Tampoco se habla de si existe hiperproducción hormonal ya que suelen ser los tumores productores de prolactina y hormona de crecimiento los que suelen presentar más episodios apoplécticos. Agradeceríamos la publicación de su amplia experiencia en el abordaje de estas lesiones y sugerimos un manejo conjunto con endocrinólogos con especial dedicación a la neuroendocrinología.

C. Páramo Neuroendocrinóloga Complejo Hospitalario Universitario de Vigo 